\title{
On-Site Determination and Monitoring of Real-Time Fluence Delivery for an Operating UV Reactor Based on a True Fluence Rate Detector
}

\author{
Mengkai Li, ${ }^{\dagger,}$ Wentao Li, ${ }^{\dagger}$ Zhimin Qiang, ${ }^{* \dagger}$ and Ernest R. Blatchley, III*,\$, ${ }^{*}$ \\ ${ }^{\dagger}$ Laboratory of Drinking Water Science and Technology, Research Center for Eco-Environmental Sciences, University of Chinese \\ Academy of Sciences, Chinese Academy of Sciences, 18 Shuang-qing Road, Beijing 100085, China \\ ${ }^{\ddagger}$ Lyles School of Civil Engineering, Purdue University, 550 Stadium Mall Drive, West Lafayette, Indiana 47907, United States \\ ${ }^{\S}$ Division of Environmental \& Ecological Engineering, Purdue University, West Lafayette, Indiana 47907, United States
}

Supporting Information

\begin{abstract}
At present, on-site fluence (distribution) determination and monitoring of an operating UV system represent a considerable challenge. The recently developed microfluorescent silica detector (MFSD) is able to measure the approximate true fluence rate (FR) at a fixed position in a UV reactor that can be compared with a FR model directly. Hence it has provided a connection between model calculation and real-time fluence determination. In this study, an on-site determination and monitoring method of fluence delivery for an operating UV reactor was developed. True FR detectors, a UV transmittance (UVT) meter, and a flow rate meter were used for fundamental measurements. The fluence distribution, as well as reduction equivalent fluence $(\mathrm{REF}), 10$ th percentile dose in the UV fluence distribution $\left(F_{10}\right)$, minimum fluence $\left(F_{\text {min }}\right)$, and mean fluence $\left(F_{\text {mean }}\right)$ of a test reactor, was calculated in advance by the combined use of computational fluid dynamics and FR field modeling. A field test was carried out on the test reactor for disinfection of a secondary water supply. The estimated realtime REF, $F_{10}, F_{\text {min }}$, and $F_{\text {mean }}$ decreased $73.6 \%, 71.4 \%, 69.6 \%$, and $72.9 \%$, respectively, during a 6-month period, which was attributable to lamp output attenuation and sleeve fouling. The results were analyzed with synchronous data from a previously developed triparameter UV monitoring system and water temperature sensor. This study allowed demonstration of an accurate method for on-site, real-time fluence determination which could be used to enhance the security and public confidence of UVbased water treatment processes.

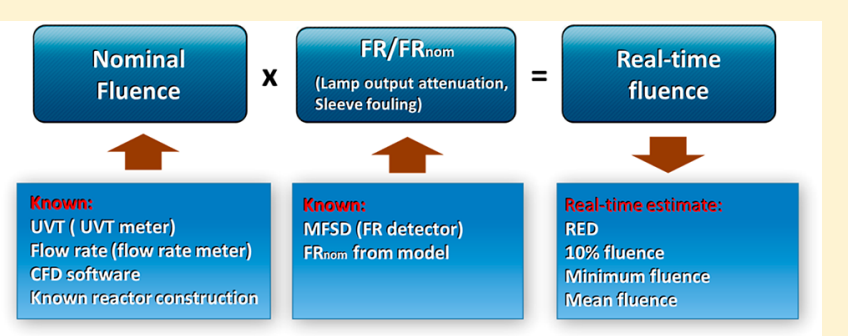

\section{INTRODUCTION}

Ultraviolet (UV) processes have been widely used for disinfection and chemical oxidation in water and wastewater treatment. A considerable body of research has addressed the kinetics and mechanisms of disinfection and oxidation, ${ }^{1-5}$ as well as the development of efficient reactor designs. ${ }^{6-8}$ Development and implementation of real-time UV reactor monitoring devices are also important for successful application of UV technology, because UV reactor performance can be affected by several factors, including lamp output, sleeve fouling, UV transmittance (UVT) of water, and flow rate. ${ }^{9-11}$ On-site determination and long-term monitoring of fluence delivery are important components for security assurance of operating UV reactors.

The performance of a UV reactor is governed by the UV fluence or, more precisely, by the fluence distribution. The initial (nominal) fluence determination (as measured by biodosimetry) of an ex-factory UV reactor has been investigated by many researchers. ${ }^{12-17}$ Fluence estimation during subsequent operation is often based on initial fluence and monitoring of flow rate, UVT, and conventional detector readings. Monitoring protocols based on this approach have been proposed and applied. ${ }^{18-20}$
Reactor validation and monitoring by this approach suffers from several important drawbacks. First, biodosimetry is usually conducted by a third party, using instruments that are different than those that will be used to monitor reactor performance. Second, biodosimetry provides only a measure of the reduction equivalent fluence (REF), a nominal measure of fluence that does not account for the fluence distribution. Third, biodosimetric methods are based on challenge microbes that are different from those that are the target of actual treatment applications; unless the kinetics of inactivation (i.e., fluenceresponse behavior) is identical for challenge and target microbes, the results of biodosimetry experiments cannot be used to predict reactor performance, in terms of inactivation of the target microbe. Fourth, in some developing countries, standards for quality control in biodosimetry have not yet been established or are difficult to enforce. In the absence of such standards, the results of biodosimetric assays may also be questioned.

Received: March 8, 2017

Revised: June 19, 2017

Accepted: June 26, 2017

Published: June 26, 2017 


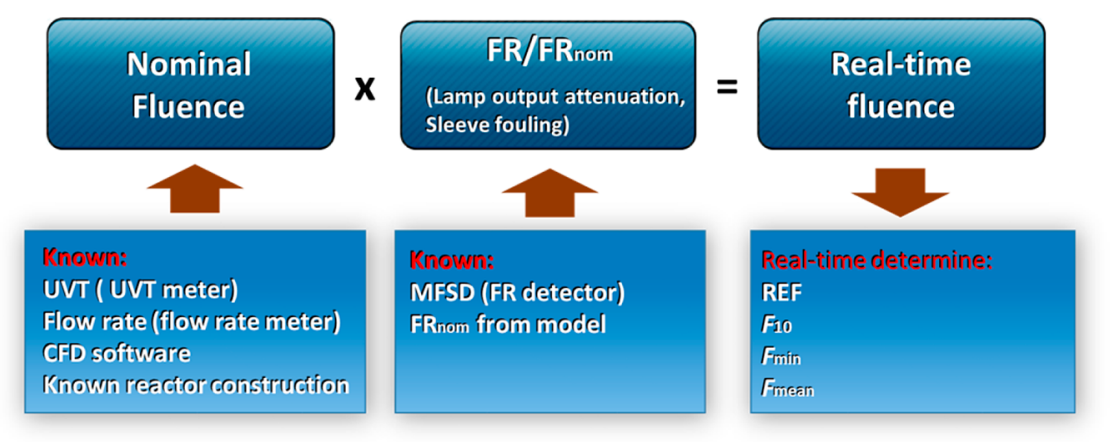

Figure 1. Schematic illustration of the on-site fluence estimation and monitoring method.

Recently, a microfluorescent silica detector (MFSD) was developed which has the ability to capture and detect photons from essentially any direction. ${ }^{21,22}$ As such, it is an approximate true fluence rate (FR) detector. For on-site fluence determination and monitoring, this aspect of MFSD performance facilitates comparisons between the detector reading and model simulation. Therefore, the MFSD signal can provide a "bridge" between model calculations and on-site fluence determination. Because computational fluid dynamics (CFD) coupled with FR distribution modeling (i.e., CFD-I) has been widely applied in fluence (distribution) evaluation of UV reactors, ${ }^{13}$ it was hypothesized that the combination of the MFSD and CFD-I simulations could be used for on-site, realtime monitoring of fluence distribution delivery.

This paper presents an on-site monitoring method of fluence delivery for an operating UV reactor. The method is based on measurements from an MFSD, a flow rate meter, and a UV transmittance (UVT) meter, coupled with CFD-I simulations. A field test based on this approach was carried out to examine applicability of this method in practical application. This simple, low-cost method offers the potential for on-site inspection and long-term monitoring of UV reactors.

\section{EXPERIMENTAL SECTION}

Method Description. For an operating UV reactor, realtime fluence is primarily affected by four variables: flow rate (Q), UVT, real-time lamp output power, and sleeve fouling. So in general, through on-site measurement of these four variables, real-time fluence (distribution) delivery can be calculated using model calculations (combined simulations of optical and fluidic fields) with a known, fixed reactor geometry. A schematic illustration of the fluence delivery estimation method is presented in Figure 1.

Real-time flow rate and UVT can be reliably measured using off-the-shelf instruments. However, real-time measurements of lamp output power and sleeve fouling are more difficult. For this study, uniform lamp output and quartz sleeve fouling along the longitudinal lamp axis were assumed. Based on these assumptions, real-time lamp output power and sleeve fouling can be described by a lamp output attenuation coefficient $(N$, i.e., the ratio of real-time lamp output power to nominal lamp output power) and a sleeve fouling attenuation coefficient $(F$, i.e., $F=1.00$ when the sleeve was absolutely clean), respectively. Hence, real-time fluence delivery is linearly dependent on the lamp output attenuation coefficient and sleeve fouling attenuation coefficient

$$
F_{\mathrm{RT}}(Q, \mathrm{UVT})=F_{\text {nom }}(Q, \mathrm{UVT}) \cdot N \cdot F
$$

where $F_{\mathrm{RT}}(Q, \mathrm{UVT})$ and $F_{\text {nom }}(Q, \mathrm{UVT})$ are the real-time and nominal fluence deliveries (i.e., the nominal fluence calculated by CFD-I in the condition of $N=1$ and $F=1$ ) of a UV reactor at a given $Q$ and UVT, respectively. In addition, since FR variation at a given point is linearly dependent on lamp output and sleeve fouling variations if UVT remains constant, the ratio of measured real-time $\mathrm{FR}\left(\mathrm{FR}_{\mathrm{RT}}\right)$ at a test point to the modeled FR at nominal lamp output power $\left(\mathrm{FR}_{\text {nom }}\right)$ should be equal to the product of $N$ and $F$ as follows:

$$
\frac{\mathrm{FR}_{\mathrm{RT}}(\mathrm{UVT})}{\mathrm{FR}_{\text {nom }}(\mathrm{UVT})}=N \cdot F
$$

Following this logic, real-time fluence could be acquired by

$$
F_{\mathrm{RT}}(Q, \mathrm{UVT})=F_{\text {nom }}(Q, \mathrm{UVT}) \cdot \frac{\mathrm{FR}_{\mathrm{RT}}(\mathrm{UVT})}{\mathrm{FR}_{\text {nom }}(\mathrm{UVT})}
$$

Therefore, by measuring UVT, flow rate, and FR at a fixed location, it was hypothesized that real-time fluence could be estimated by integration with model simulation results. In practical applications, a true FR detector (e.g., MFSD) can be positioned in an operating reactor to allow on-site, real-time fluence determination. If the true FR detector is fixed at a given location in the reactor, long-term fluence monitoring could be achieved.

A series of CFD-I simulations were conducted to complement the measurements from the field experiment. Because of CPU time requirements to complete CFD-I simulations, realtime calculations of fluence distributions are not possible at present. As a surrogate for this information, four indicators (i.e., reduction equivalent fluence (REF), 10th percentile dose in the UV fluence distribution $\left(F_{10}\right)$, minimum fluence $\left(F_{\min }\right)$, and mean fluence $\left.\left(F_{\text {mean }}\right)\right)$ as functions of UVT and $Q$ for a baseline condition (i.e., $N \equiv 1.00$ and $F \equiv 1.00$ ) were calculated in advance. For application in a large-scale UV system containing multiple lamps, the same initial and real-time $N$ and $F$ of each lamp/sleeve could be assumed. Multiple test positions (for onsite determination) or multiple detectors (for monitoring) could be applied at appropriate positions to represent the operating status of lamps as many as possible.

Model Simulation. The FR and fluence delivery calculations were accomplished with a commercial CFD software package (ANSYS Fluent 13.0, Canonsbury, PA, USA). The realizable $k-\varepsilon$ model and discrete ordinates (DO) model were employed for the fluidic and optical (FR distribution) simulations, respectively. The principal parameters of the DO model were described in Text S1. Simulations followed a Lagrangian approach in which 5000 particles were uniformly released at the cross-section of the inlet, and their 
trajectories were simulated using a discrete phase model. Integration of CFD results with fluence rate field modeling results was conducted for UVT values of $97.5 \%, 95 \%, 92.5 \%$, $90 \%, 87.5$, and $85 \%$. The matrix of simulation results also included flow rates of $0.25,0.5,0.75,1,1.5$, and $2 \mathrm{~m}^{3} / \mathrm{h}$. Collectively, these values of UVT and flow rate spanned the range of operating conditions observed in the field experiment (see the description below).

Test Detector. The MFSD has been demonstrated for in situ measurements of FR distributions in UV reactors. ${ }^{22,23}$ Cylindrical Ge-doped silica ( $0.3 \mathrm{~mm}$ diameter, $1.0 \mathrm{~mm}$ length) was used as the photosensitive material, which was able to capture photons from nearly all directions with a maximum angle error of $3 \% .{ }^{18}$ The UV-excited fluorescence was then transmitted through an optical fiber $(0.3 \mathrm{~mm}$ diameter, $400 \mathrm{~mm}$ length) to a photodiode (BPW 34B silicon PIN photodiode) and digitally displayed and collected by a recorder (R2001X, Pangu Co. China).

Field Test. To examine the central hypothesis of this research, a field test was carried out using a single-lamp, annular stainless steel UV reactor (inner diameter $140 \mathrm{~mm}$, inner length $950 \mathrm{~mm}$ ) which was applied for disinfection of a secondary water supply (SWS) in a residential community located in Zhengzhou City of Henan Province, China (Figure 2). This

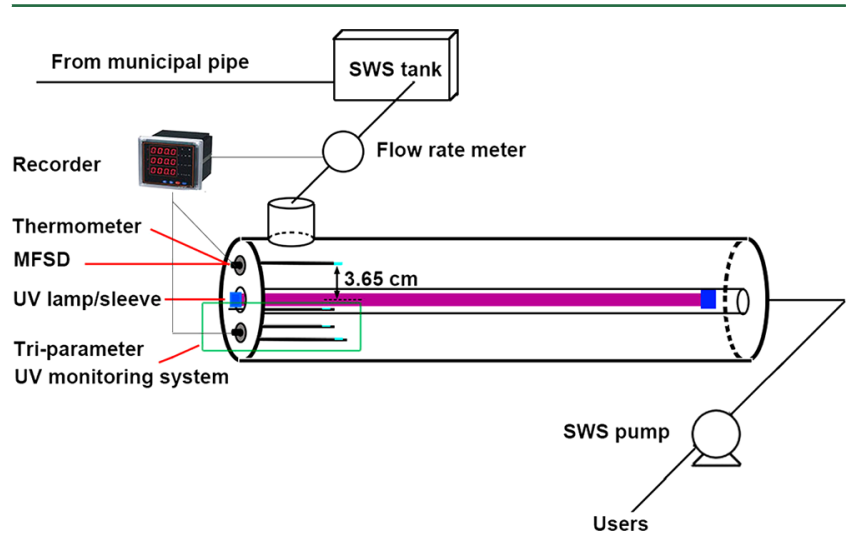

Figure 2. Schematic illustration of the UV disinfection of the secondary water supply for the field test.

community includes 250 apartments. Treated water from municipal distribution pipes flowed into a storage tank, after which it was disinfected by the UV reactor and then pumped to user taps.

The MFSD was placed at a fixed location in the reactor during the on-site fluence determination experiment. The UV reactor contained a low-pressure high-output amalgam lamp (Xiashi Wanhua Co., China; arc length $786 \mathrm{~mm}$, electric power $105 \mathrm{~W}$, nominal UVC efficiency $32 \%$ ) centered inside a quartz sleeve $(23 \mathrm{~mm}$ o.d.). A flow rate meter was placed between the reactor inlet and storage tank. A previously developed triparameter UV monitoring system (TPUM) ${ }^{9}$ was installed in the UV reactor which allowed real-time measurement of UVT. An MFSD (length $150 \mathrm{~mm}$, outside diameter $8 \mathrm{~mm}$ ) was used as the test FR detector, which was fixed on a flange parallel to the lamp at a radial distance $=36.5 \mathrm{~mm}$ and axial distance to the lamp electrode $\approx 100 \mathrm{~mm}$. In addition, water temperature was monitored by a PT100 thermal resistance thermometer. The real-time signals of flow rate, UVT, FR, and water temperature were simultaneously recorded by a recorder (R2001X, Pangu Co. China) with a time interval of $4 \mathrm{~min}$.

\section{RESULTS AND DISCUSSION}

FR at the Test Point. Figure 3 illustrates the FR distribution at the central cross-section of the test reactor, as

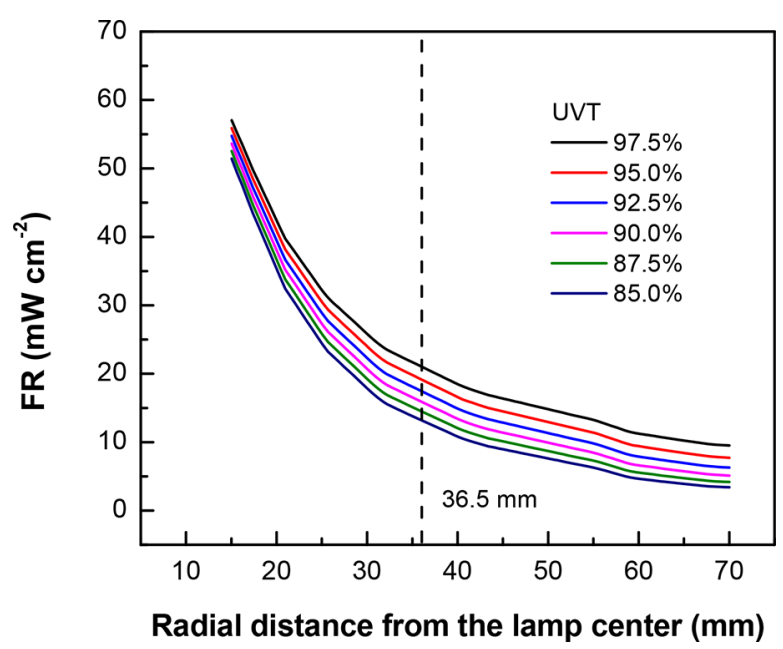

Figure 3. Radial FR distributions from DO model simulation at various UVT values (vertical, dashed line indicates the position of the MFSD in this study).

calculated by the DO model for the UVT range of 97.5-85.0\%. The FR decreased on approach to the reactor wall; UVT reduction attenuated the entire FR distribution profile. These data were also used for real-time $N \times F$ estimation by eq 2 . Note that both experimental and modeled results in previous studies have demonstrated that the axial FR distribution remains nearly constant along the length of the lamp for locations that are more than $50 \mathrm{~mm}$ from the lamp end. ${ }^{22,24}$ So the radial FR distribution at the central cross-section was regarded as being representative of the cross-section where the test detector (i.e., MFSD) was placed (about $100 \mathrm{~mm}$ axial distance to the lamp electrode). Hence, $\mathrm{FR}_{\text {nom }}$ at the detector location was estimated as a function of UVT by means of an exponential regression analysis based on the six FR values at various UVTs which had a radial distance of $36.5 \mathrm{~mm}$ to lamp center:

$$
\mathrm{FR}_{\text {nom }}(\mathrm{UVT})=22.6 e^{-7.89 \log _{10}(\mathrm{UVT})}
$$

The $R^{2}$ value for this function was greater than $99.9 \%$. Therefore, eq 4 can be used to provide an accurate $F_{\text {nom }}$ value for eq 2 based on a real-time UVT value (measured by UVT meter).

Fluence Distribution Calculation. The trajectories of 5000 particles were simulated in the CFD-I calculation process; resulting fluence distributions for the flow rate range of $0.25-$ $2.00 \mathrm{~m}^{3} \mathrm{~h}^{-1}$ and UVT range of $85.0-97.5 \%$ are illustrated in Figure 4. The minimum fluence (dose) received by the simulation particles increased with decreasing flow rate for each UVT condition. On the other hand, with the lower UVT value, more particles received lower fluence, thereby contributing to poorer disinfection performance.

Based on the calculated fluence distributions, four indicators of reactor performance were defined: REF, $F_{10}, F_{\text {min }}$, and $F_{\text {mean }}$, for each combination of flow rate and UVT (see Figures S1S4). Nonlinear regression analysis of REF as functions of UVT and flow rate was conducted and shown in eq 5 with $R^{2}=$ 0.994 . 


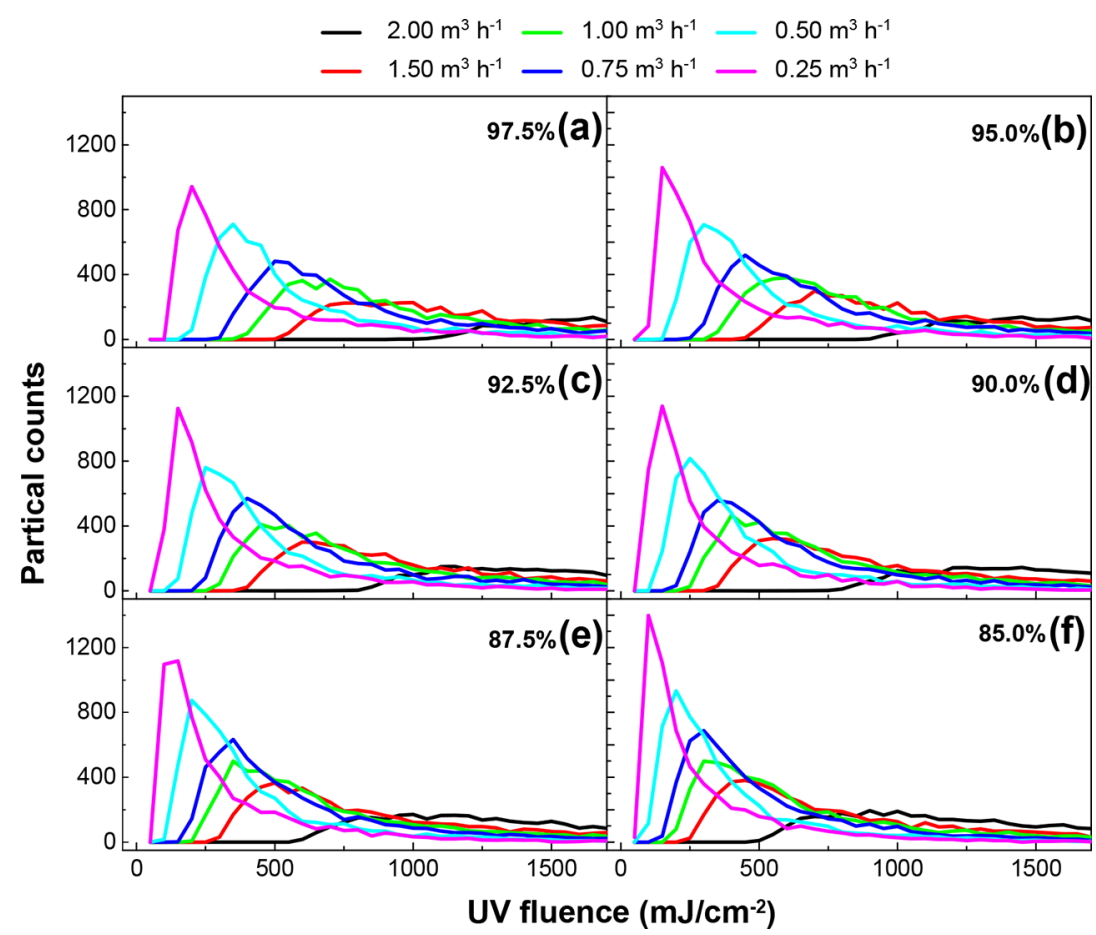

Figure 4. Calculated fluence distributions of the test UV reactor at UV transmittances of $97.5 \%$ (a), $95.0 \%$ (b), $92.5 \%$ (c), $90.0 \%$ (d), $87.5 \%$ (e), and $85.0 \%$ (f). Numerical values in the upper-right corner of each panel indicate $\mathrm{UVT}_{254}$.

$$
\operatorname{REF}_{\text {nom }}(Q, U V T)=\left[2317.3 \log _{10}(U V T)+301.4\right] Q^{-0.944}
$$

Therefore, by using eq 5 , the nominal REF value could be rapidly estimated by interpolation.

Using a similar approach, $F_{10}, F_{\min }$ and $F_{\text {mean }}$ for the baseline condition were estimated by use of eqs $6-8$ based on the data in Figures $\mathrm{S} 2-\mathrm{S} 4$ :

$$
\begin{aligned}
& F_{10, \text { nom }}(Q, \mathrm{UVT})=\left[2984.5 \log _{10}(\mathrm{UVT})+386\right] Q^{-1.046} \\
& F_{\text {mean,nom }}(Q, \mathrm{UVT})=\left[5906.6 \log _{10}(\mathrm{UVT})+1009.5\right] Q^{-1.07} \\
& F_{\text {min,nom }}(Q, \mathrm{UVT})=\left[2225.6 \log _{10}(\mathrm{UVT})+257.9\right] Q^{-1.08}
\end{aligned}
$$

All $R^{2}$ values of the regression processes were higher than $99.3 \%$, which suggested high accuracy of the interpolation functions. It is important to emphasize that these functions were intended for interpolation (not extrapolation) among measured values. Moreover, these expressions are valid only for the reactor system that was employed in the field experiments described in this work. Similar expressions could be developed for other reactor systems following an analogous approach.

Parameter Determinations. A field experiment was carried out in which an MFSD was fixed in the UV reactor. As indicated above, the reactor system was also fitted with instruments to allow real-time measurements of flow rate and $\mathrm{UVT}_{254}$. The results of continuous measurements of FR, flow rate, and $\mathrm{UVT}_{254}$ for the 6-month period of this experiment are illustrated in Figure 5. Figure 5a illustrates that measured FR decreased gradually from 16.85 to $2.45 \mathrm{~mW} \cdot \mathrm{cm}^{-2}$ during the measurement period. Because UVT demonstrated minimal variation in this period (Figure 5c), the changes in fluence were likely attributable to attenuation of lamp output (i.e., lamp

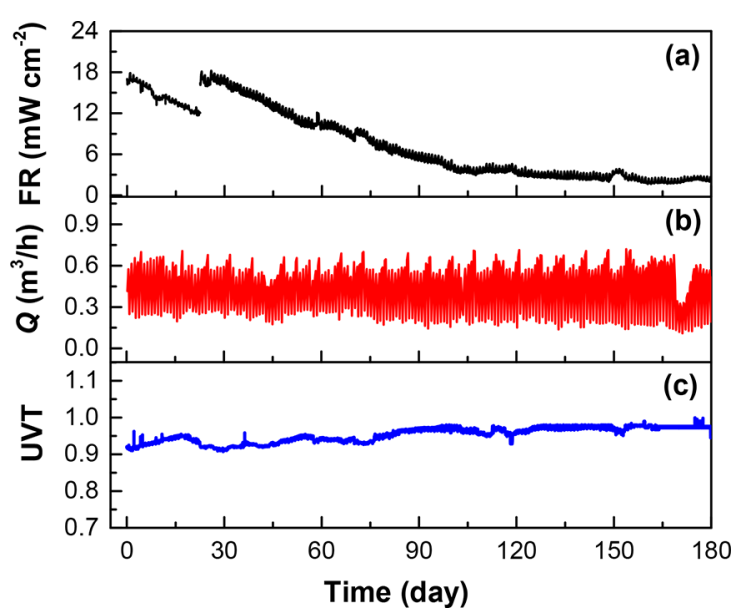

Figure 5. Real-time MFSD reading, FR (a), flow rate, $Q$ (b), and UVT values $(\mathrm{c})$ during the 6-month operation period.

aging) and sleeve fouling. Because no sleeve cleaning system was installed in this reactor, sleeve fouling occurred progressively through the length of the study period. Moreover, the MFSD reading was also affected by lamp aging and water temperature. In fact, water temperature variation from August (summer) to February (winter) in Zhengzhou could greatly impact the $\mathrm{Hg}$ vapor pressure inside the lamp, which would also affect lamp output. Collectively, these factors contributed to an $85.5 \%$ attenuation on the FR value at the test point over the course of this experiment. In addition, the FR displayed a sudden increase on the 22nd day of the test (see Figure 5a). This behavior was attributable to a sleeve maintenance. The onsite engineers stopped the pump and cut off the power, and then the sleeve was taken out and cleaned with a brush. Hence it resulted in an increase of UV transmittance of the quartz sleeve. 
Figure $5 \mathrm{~b}$ illustrates that the flow rate displayed daily periodic fluctuations in the range of $0.15-0.7 \mathrm{~m}^{3} \mathrm{~h}^{-1}$ during the test period. This characteristic of SWS systems is dependent on the daily water use patterns of residents living in the community. Previous investigations have shown that the flow rate typically reaches a maximum value between 8 and $11 \mathrm{pm}$ when most people cook dinner and take showers. ${ }^{19}$ The minimum flow rate is found in the early morning hours when people are asleep. No obvious differences in flow rate were observed between summer and winter in this community.

$\mathrm{UVT}_{254}$ displayed a slight increase over the period of this experiment (Figure 5c), which may have been the result of changes in source water quality in Zhengzhou from summer to winter. A higher $\mathrm{UVT}_{254}$ will enhance disinfection performance. It is relevant to note that by following this mode of continuous monitoring of critical input parameters, it is possible to use these data to define alarm conditions for the UV disinfection system.

On-Site Fluence Determination. Through real-time measurements of $\mathrm{FR}, \mathrm{UVT}_{254}$, and flow rate, the four indicators of the fluence delivery for the test UV reactor at any time during the 6-month operation were calculated (eqs 3, 5-8) and shown in Figure 6, implying that real-time and on-site

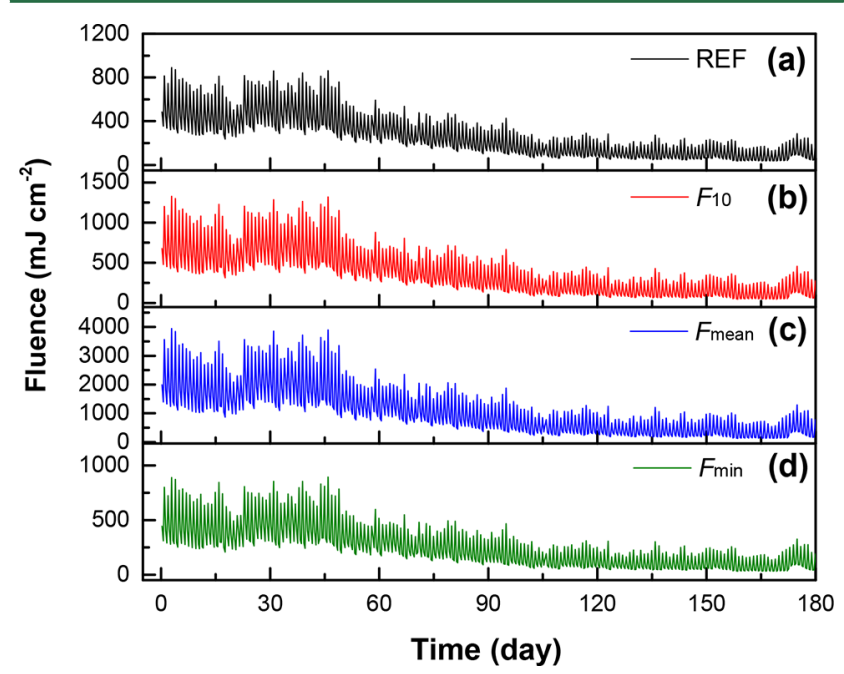

Figure 6. Real-time estimated values of reduction equivalent fluence (a), 10th percentile dose in the UV fluence distribution (b), mean fluence (c), and minimum fluence (d) during the 6-month operation period.

estimation of fluence delivery could be implemented using the true FR detector (MFSD), UVT meter, and flow rate meter without biodosimetry. For this experiment, all four indicators decreased according to a similar trend, which was caused by sleeve fouling and lamp output attenuation. The daily average REF, $F_{10}$, and $F_{\min }$ dropped $73.6 \%, 71.4 \%$, and $69.6 \%$, respectively, indicating a large attenuation on the disinfection efficiency for this running reactor. Likewise, a $72.9 \%$ drop of $F_{\text {mean }}$ was observed, similarly implying an efficiency decrease for this reactor. It should be noted that $F_{\text {mean }}$ is not a good indicator of disinfection efficacy in UV photoreactors but may be relevant for estimation of process efficiency in applications of UV photoreactors for direct photolysis or advanced oxidation processes, where reactant conversion is typically much smaller than in disinfection systems. In addition, the periodic changes of flow rate (in Figure 5b) induced a synchronous fluctuation of fluence delivery. Similar results were also obtained from the other monitoring method (with a nonFR UV detector) for SWS UV disinfection in a previous study. ${ }^{19}$

Estimation of $\boldsymbol{N}$ and $\boldsymbol{F}$ with Triparameter Monitoring System (TPUM). In the field test, a water temperature detector and a previously developed TPUM were also installed in the test reactor; data from these instruments were used to test results from the newly developed monitoring method. The monitoring results of $N$ (lamp out attenuation coefficient) and $F$ (lamp fouling attenuation coefficient) from the TPUM during the 6-month are shown in Figures $7 \mathrm{a}$ and $7 \mathrm{~b}$,

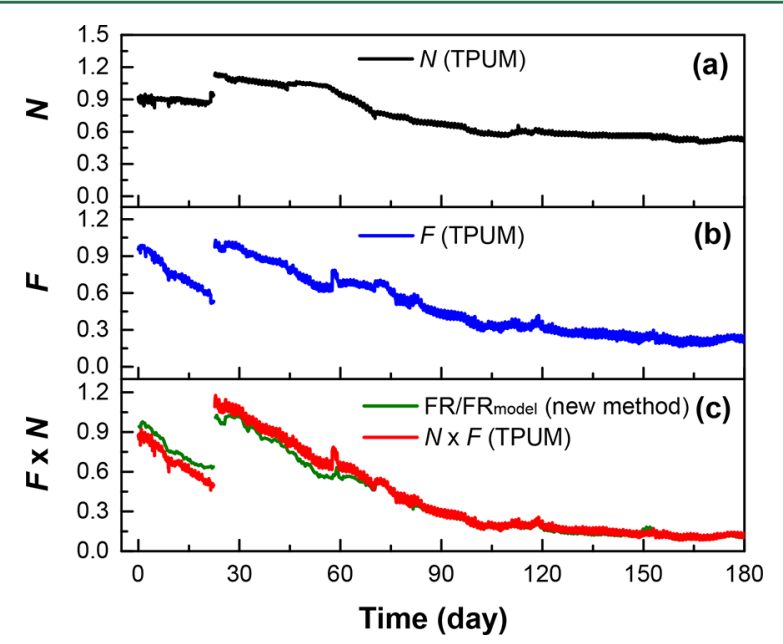

Figure 7. Monitored lamp output attenuation coefficient (a), sleeve fouling attenuation coefficient (b) from triparameter UV monitoring system (TPUM), and real-time $N \times F$ data from the TPUM and the method developed by this study (c).

respectively. In general, $N$ decreased to about 0.544 over the 6-month operation of the system, which principally arose from the effects of lamp aging and water temperature, as discussed above. For comparison, an attenuation coefficient of 0.8 for 1 year operation time was reported by the manufacturer. Moreover, the water temperature decreased from 24.8 to 9.3 ${ }^{\circ} \mathrm{C}$ (see Figure S5), which could also attenuate lamp output. As described above, sleeve maintenance was carried out on the 22 nd day of the experiment. It is believed that shaking of the lamp-body during the maintenance process resulted in movement of the amalgam point, such that the mercury vapor pressure inside the lamp chamber changed to a better working status so that $N$ increased after the maintenance.

$F$ decreased to 0.240 at the end of the 6-month experiment. With the same water supply (i.e., municipal water supply of Zhengzhou, China), our previous study also reported that a 6month operating period yielded a similar fouling coefficient (0.30) in a UV reactor. ${ }^{19}$ After sleeve maintenance on the $22 \mathrm{nd}$ day, $F$ returned to the initial condition and then gradually reduced to 0.78 by the 44th day (another 22-day operation).

Eq 2 indicates that the product $N \times F$ could also be measured by the ratio of real-time to nominal true FR detector readings. Hence, a comparison of $N \times F$ obtained from the TPUM and eq 2 (i.e., methodology in this study) is shown in Figure $7 \mathrm{c}$. The two curves have similar profiles during the 6month operation time, which supports the validity of the $N \times F$ estimation method from eq 2 . The results also show that the absolute values at each time value of two curves are nearly 
identical. In fact, this is unexpected because the $N$ determined by the TPUM was normalized to a value of 1.00 as an initial condition, while the FR model calculation in eq 2 used the nominal lamp output efficiency value (i.e., 32.5\%) without any normalization on lamp output in initial condition. If the nominal lamp efficiency (i.e., 32.5\%) used for the calculation of $\mathrm{FR}_{\text {nom }}$ (UVT) in eq 2 was not accurate (i.e., accurately expressing the actual lamp efficiency at the initial condition), the absolute values of two curves in Figure $7 \mathrm{c}$ cannot be identical.

Discussion of Potential Application. CFD models (integrated with fluence rate field models) are powerful tools for estimation of fluence distribution delivery by UV reactors. To date, the application of CFD-I models has focused on simulations of fixed operating conditions (e.g., optimal design of the reactor). The development of the MFSD allows direct comparisons between measurements and FR model simulations, which provides a connection between model simulation results and on-site monitoring. Consequently, this instrument allows implementation of an on-site fluence estimation and monitoring method that can be used to provide security assurance in continuously operating UV reactors. It is also important that this method may implement a simple, low-cost approach that is likely to be particularly relevant in developing countries.

In the application of this method as a monitoring process, the detector was fixed so as to eliminate error associated with positioning. However, for an on-site fluence determination process, the positioning error could induce inaccuracy of the real-time fluence estimation. Replicate tests at a given location could be used to reduce this error. In addition, because of CPU operation time requirements, CFD software cannot be applied directly for real-time and continuous simulations of UV fluence. As a surrogate for this approach, on-site estimation or monitoring of four indicators (i.e., REF, $F_{10}, F_{\text {min }}$, and $F_{\text {mean }}$ ) was applied in this study, whose calculation relied on empirical regression equations (i.e., eqs 5-8).

Two assumptions (uniform lamp output and uniform sleeve fouling condition along the sleeve) have been applied in this study that could represent sources of error in this monitoring approach. To address these limitations, measurements of output distribution along the lamp and sleeve fouling distribution will be carried out in the future. Then, axial correction factors could be incorporated into this method, and the detector position could be optimized to reduce the error introduced by these two assumptions.

\section{ASSOCIATED CONTENT}

\section{S Supporting Information}

The Supporting Information is available free of charge on the ACS Publications website at DOI: 10.1021/acs.est.7b01241.

Estimates of REF (Figure S1), $F_{10}$ (Figure S2), $F_{\text {min }}$ (Figure S3), and $F_{\text {mean }}$ (Figure S4) based on CFD simulations, as well as their exponential regression curves as a function of flow rate at various UVT values; variation of water temperature during the 6-month operation period (Figure S5) (PDF)

\section{AUTHOR INFORMATION}

\section{Corresponding Authors}

*Phone: 1-765-494-0316. Fax 1-765-494-0395. E-mail: blatch@ purdue.edu. Corresponding author address: Lyles School of
Civil Engineering, Purdue University, 550 Stadium Mall Drive, West Lafayette, IN 47907, USA (E.R.B.).

*E-mail: qiangz@rcees.ac.cn (Z.Q.).

ORCID

Ernest R. Blatchley III: 0000-0002-4561-8635

Notes

The authors declare no competing financial interest.

\section{ACKNOWLEDGMENTS}

The authors gratefully acknowledge financial support from the National Natural Science Foundation of China (51408592, $516115400344,21590814)$, the National Key Research and Development Project of China (2016YFC0400802), and the Edward M. Curtis Visiting Professorship of Purdue University.

\section{REFERENCES}

(1) Beck, S. E.; Wright, H. B.; Hargy, T. M.; Larason, T. C.; Linden, K. G. Action spectra for validation of pathogen disinfection in medium-pressure ultraviolet (UV) systems. Water Res. 2015, 70, 2737.

(2) Xiang, Y.; Fang, J.; Shang, C. Kinetics and pathways of ibuprofen degradation by the UV/chlorine advanced oxidation process. Water Res. 2016, 90, 301-308.

(3) Mestankova, H.; Parker, A. M.; Bramaz, N.; Canonica, S.; Schirmer, K.; von Gunten, U.; Linden, K. G. Transformation of Contaminant Candidate List (CCL3) compounds during ozonation and advanced oxidation processes in drinking water: Assessment of biological effects. Water Res. 2016, 93, 110-120.

(4) Fang, J. Y.; Fu, Y.; Shang, C. The roles of reactive species in micropollutant degradation in the UV/free chlorine system. Environ. Sci. Technol. 2014, 48 (3), 1859-1868.

(5) Song, K.; Mohseni, M.; Taghipour, F. Application of ultraviolet light-emitting diodes (UV-LEDs) for water disinfection: A review. Water Res. 2016, 94, 341-349.

(6) Elyasi, S.; Taghipour, F. Simulation of UV photoreactor for degradation of chemical contaminants: model development and evaluation. Environ. Sci. Technol. 2010, 44 (6), 2056-2063.

(7) Zazueta, A. L. L.; Destaillats, H.; Li Puma, G. Radiation field modeling and optimization of a compact and modular multi-plate photocatalytic reactor (MPPR) for air/water purification by Monte Carlo method. Chem. Eng. J. 2013, 217, 475-485.

(8) Bolton, J. R.; Mayor-Smith, I.; Linden, K. G. Rethinking the concepts of fluence (UV dose) and fluence rate: The importance of photon-based units - a systemic review. Photochem. Photobiol. 2015, 91 (6), 1252-1262.

(9) Qiang, Z. M.; Li, M. K.; Bolton, J. R. Development of a triparameter online monitoring system for UV disinfection reactors. Chem. Eng. J. 2013, 222, 101-107.

(10) Wait, I. W.; Blatchley, E. R., III Model of radiation transmittance by inorganic fouling on UV reactor lamp sleeves. Water Environ. Res. 2010, 82 (11), 2272-2278.

(11) Wait, I. W.; Johnston, C. T.; Blatchley, E. R., III The influence of oxidation reduction potential and water treatment processes on quartz lamp sleeve fouling in ultraviolet disinfection reactors. Water Res. 2007, 41 (11), 2427-2436.

(12) Blatchley, E. R., III; Shen, C. Y.; Naunovic, Z.; Lin, L. S.; Lyn, D. A.; Robinson, J. P.; Ragheb, K.; Gregori, G.; Bergstrom, D. E.; Fang, S. Y.; Guan, Y. H.; Jennings, K.; Gunaratna, N. Dyed microspheres for quantification of UV dose distributions: Photochemical reactor characterization by Lagrangian actinometry. J. Environ. Eng. 2006, 132 (11), 1390-1403.

(13) Blatchley, E. R., III; Shen, C.; Scheible, O. K.; Robinson, J. P.; Ragheb, K.; Bergstrom, D. E.; Rokjer, D. Validation of large-scale, monochromatic UV disinfection systems for drinking water using dyed microspheres. Water Res. 2008, 42 (3), 677-688.

(14) Li, M. K.; Qiang, Z. M.; Wang, C.; Bolton, J. R.; Lian, J. F. Development of monitored tunable biodosimetry for fluence 
validation in an ultraviolet disinfection reactor. Sep. Purif. Technol. 2013, 117, 12-17.

(15) Qiang, Z. M.; Li, M. K.; Bolton, J. R; Qu, J. H.; Wang, C. Estimating the fluence delivery in UV disinfection reactors using a 'detector-model' combination method. Chem. Eng. J. 2013, 233, 3946.

(16) Templeton, M. R.; Hofmann, R.; Andrews, R. C.; Whitby, G. E. Biodosimetry testing of a simplified computational model for the UV disinfection of wastewater. J. Environ. Eng. Sci. 2006, 5 (1), 29-36.

(17) Jin, S.; Mofidi, A. A.; Linden, K. G. Polychromatic UV fluence measurement using chemical actinometry, biodosimetry, and mathematical techniques. J. Environ. Eng. 2006, 132 (8), 831-841.

(18) Li, M. K.; Qiang, Z. M.; Bolton, J. R.; Li, W. T.; Wang, C. Improved method for real-time fluence monitoring in UV reactors. $J$. Environ. Eng. 2015, 141 (4), 04014075.

(19) Li, M. K.; Qiang, Z. M.; Bolton, J. R.; Li, W. T.; Chen, P. UV disinfection of secondary water supply: Online monitoring with microfluorescent silica detectors. Chem. Eng. J. 2014, 255, 165-170.

(20) Bolton, J. R.; Wright, H.; Rokjer, D. Using a mathematical fluence rate model to estimate the sensor readings in a multi-lamp ultraviolet reactor. J. Environ. Eng. Sci. 2005, 4 (S1), S27-S31.

(21) Li, M. K.; Qiang, Z. M.; Bolton, J. R.; Ben, W. W. Impact of reflection on the fluence rate distribution in a UV reactor with various inner walls as measured using a micro-fluorescent silica detector. Water Res. 2012, 46 (11), 3595-3602.

(22) Li, M. K.; Qiang, Z. M.; Li, T. G.; Bolton, J. R.; Liu, C. L. In situ measurement of UV fluence rate distribution by use of a micro fluorescent silica detector. Environ. Sci. Technol. 2011, 45 (7), 30343039.

(23) Li, M. K.; Qiang, Z. M.; Bolton, J. R. In situ detailed fluence rate distributions in a UV reactor with multiple low-pressure lamps: Comparison of experimental and model results. Chem. Eng. J. 2013, $214,55-62$.

(24) Liu, D.; Wu, C.; Linden, K. G.; Ducoste, J. Numerical simulation of UV disinfection reactors: Evaluation of alternative turbulence models. Appl. Math. Model. 2007, 31 (9), 1753-1769. 